\title{
WAVE SCATTERING AT A TAPERED FREE END OF AN ELASTIC PLATE
}

\author{
YAgOUB N. Al-NASSAR AND MUHAMMAd A. HAWWA \\ Department of Mechanical Engineering, \\ King Fahd University of Petroleum and Minerals \\ Dhahran 31261, Saudi Arabia. \\ ynassar@kfupm.edu.sa
}

\begin{abstract}
This paper focuses on the interaction of $\mathrm{SH}$ elastic waves in a plate with a tapered free end. The plate is modeled as a semi-infinite elastic structure, which was assumed to have traction-free surfaces. The results of the analysis based on mode matching are presented for various combinations of normalized frequencies and angles of free end inclination. The reported observations form important guidelines for the interpretation of experimental data when using horizontally polarized wave as a mean for nondestructive evaluation of elastic plates.
\end{abstract}

ABSTRAK: Kertas kerja ini adalah khusus berkenaan interaksi gelombang anjal SH di dalam plat yang mempunyai hujung bebas yang tirus. Plat ini dimodelkan sebagai satu struktur elastik separa tak terhingga, yang diandaikan mempunyai permukaan nirgeseran. Keputusan analisis berdasarkan mod pemadanan dibentangkan untuk pelbagai kombinasi frekuensi ternormal dan sudut kecondongan hujung bebas. Pemerhatian yang dibentangkan memberi panduan penting dalam menginterpretasi data eksperimental penggunaan gelombang berkutub mendatar sebagai satu kaedah penilaian tanpa musnah plat anjal.

KEYWORDS: elastic plates; ultrasonic wave scattering; nondestructive evaluation

\section{INTRODUCTION}

The non-destructive testing (NDT) of materials become more important and demanding as they are used in safety critical applications such as aircraft primary structure. A decisive method for evaluating the condition of plate structures is the utilization of ultrasonic wave scattering due to the presence of inclusion or inclusions and defects such as cracks and flaws.

Methods of solution of scattering problem vary from classical approach such as mode matching and variational techniques to numerical techniques such as finite elements and boundary elements approach or combination of numerical formulation with a wave function expansion technique. There is an extensive literature on the studies of scattering of elastic waves in plates due to inclusion, cracks, or weldments. Among these, the work of Sabbagh et. al. [1] who has analyzed scattering of SH waves. In their approach, it is assumed that the plate wave guide admits a single mode only and all the higher modes in the waveguide are neglected. Abduljabbar et. al. [2] studied the diffraction of SH waves in a plate by arbitrary defects where finite element formulation and a wave function expansion technique is employed. Koshiba et al. [3] have analyzed SH wave scattering using similar approach. In their analysis, they have taken the number of normal modes and the number of nodal points 
along the interface to be independent. Datta et al. [4] and Fortunko et al. [5] studied the diffraction of SH waves by edge crack and planar defects using low -frequency and medium and long wavelength. To the author knowledge, the scattering of SH waves by an inclined edge of an elastic plate has not been reported.

Recently, an interest is raised in the reflection of elastic waves from inclined free edges of elastic plates. Wilkie-Chancellier et al. [6-8] performed finite element analyses and laser interferometer / vibrometer experiments on the reflection of the $S_{0}, A_{0}$, and A1 Lamb modes from an inclined edge of elastic plates having different values of the bevel angle. They proved that the edge mode was the resonance of different complex modes. Galán and Abascal [9] used a hybrid finite-element / boundary element technique to study Lamb mode conversion at inclined or perpendicular free edges of elastic plates for frequencies beyond the first cutoff frequency.

In this paper, a mode matching technique is used to investigate the scattering of $\mathrm{SH}$ waves due to impinging on a tapered end of an elastic plate. Mode matching procedure is followed along a fictitious boundary inside the plate while satisfying the continuity of displacement and stresses condition. A stress-free condition is satisfied at the inclined surfaces of the plate. Numerical integration leads to a system of linear algebraic equations for the reflection coefficient of the scattered field.

\section{PROBLEM FORMULATION}

Let us consider a plate shown in Fig. 1. The plate is assumed to be elastic of uniform thickness, $2 \mathrm{~h}$, and has traction-free surfaces. Two regions can be identified and these are plate region, $\Omega_{1}$, and wedge region, $\Omega_{2}$. The two regions are assumed to be separated by a fictitious boundary.

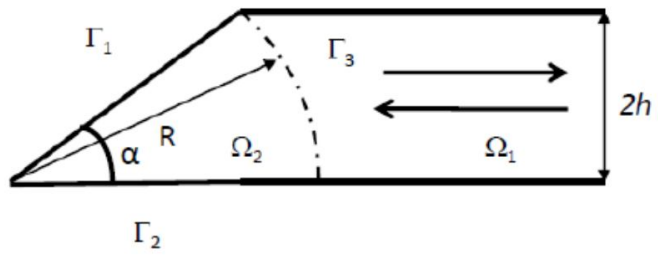

Fig. 1: Plate Geometry.

In the plate region, $\Omega_{1}$, the total displacement field, $w^{T}$, can be written as

$$
w^{T}=w^{i n c}+w^{r e f}
$$

where the superscripts inc and ref in the above equation stand for incident and reflect field, respectively. Incident field, $w^{i n c}$, consists of a single propagating mode propagating in the negative $x$-direction. The incident field is assumed to be time harmonic wave, and given by the following expression

$$
w^{i n c}=A_{i} \cos \left(\frac{i \pi y}{2 h}\right) \cdot e^{-j\left(k_{i} x-\omega t\right)}
$$


where $k$ and $\omega$ are wavenumber and frequency, respectively. The relationship between these two quantities is given by the following expression $n \pi=\sqrt{\left(\frac{2 \omega h}{c}\right)^{2}+(2 k h)^{2}}$.

The reflected field, $w^{\text {ref }}$, is generally composed of a number of reflected modes (propagating and non-propagating modes) in the plate region. The reflected field (scattered) takes the following expression

$$
w^{r e f}=\sum_{l=0}^{L} B_{l} \cos \left(\frac{l \pi y}{2 h}\right) \cdot e^{j\left(k_{l} x+\omega t\right)}
$$

The displacement field in wedge region, $\Omega_{2}$, is given by

$$
w^{t r}=\sum_{n=0}^{N} \cos \left(\frac{n \pi \theta}{\alpha}\right) \cdot\left(C_{n} J\left(\frac{n \pi}{\alpha}, \frac{\omega r}{c_{s}}\right)\right) \cdot e^{j \omega t}
$$

where $\alpha$ is the wedge angle and $c_{s}$ is the shear velocity, $(G / \rho)^{1 / 2} . G$ and $\rho$ stand for the shear modulus and density, respectively. Note that the solution in the wedge perfectly satisfies the traction free boundary conditions at the two surfaces $\Gamma_{1}$ and $\Gamma_{2}$.

Imposing the continuity of displacement and stress along the fictitious boundary, $\Gamma_{3}$, gives

$$
\begin{gathered}
\left.w^{T}\right|_{\text {plate }}=\left.w^{\text {tr }}\right|_{\text {wedge }}, r=R, 0 \leq \theta \leq \alpha \\
\left.\tau^{T}\right|_{\text {plate }}=\left.\tau^{t r}\right|_{\text {wedge }}, r=R, 0 \leq \theta \leq \alpha
\end{gathered}
$$

where $w^{t r}$ is the displacement field in the wedge. The stress field, $\tau^{T}$, is given by

$$
\left.\tau^{T}\right|_{\text {plate }}=\left.\left(\tau^{i n c}+\tau^{\text {ref }}\right)\right|_{\text {plate }}
$$

But the shear stress, $\tau$, takes the following form

$$
\tau=\tau_{x z} n_{x}+\tau_{y z} n_{y}
$$

where $n_{x}$ and $n_{y}$ are the direction cosine of the outward unit normal vector $\boldsymbol{n}$ along the boundary $\Gamma_{3}$.

It remains to choose weight functions of the form

$$
f_{w}=\cos \left(\frac{m \pi}{\alpha} \theta\right), \quad m=0,1,2, \cdots, N
$$

Then, the above boundary conditions lead to the following equations:

$$
\left.\left(\begin{array}{l}
A_{i} \cos \left(\frac{i \pi}{2 h} y\right) \cdot e^{-j k_{i} x}+ \\
\sum_{l=0}^{L} B_{l} \cos \left(\frac{l \pi}{2 h} y\right) \cdot e^{j k_{l} x}
\end{array}\right)\right|_{\substack{x=R \cos (\theta) \\
y=R \sin (\theta)}}=\left.\sum_{n=0}^{N} \cos \left(\frac{n \pi}{\alpha} \theta\right) \cdot\left(C_{n} J\left(\frac{n \pi}{\alpha}, \frac{\omega r}{c_{s}}\right)\right)\right|_{r=R}
$$




$$
\begin{aligned}
& A_{i}\left(\begin{array}{r}
-j k_{i} \cos \left(\frac{i \pi}{2 h} y\right) \cdot n_{x}- \\
\frac{i \pi}{2 h} \sin \left(\frac{i \pi}{2 h} y\right) \cdot n_{y}
\end{array}\right) \cdot e^{-j k_{i} x}+\sum_{l=0}^{L} B_{l}\left(\begin{array}{l}
j k_{l} \cos \left(\frac{l \pi}{2 h} y\right) \cdot n_{x}- \\
\frac{l \pi}{2 h} \sin \left(\frac{l \pi}{2 h} y\right) \cdot n_{y}
\end{array}\right) \cdot e^{j k_{l} x}= \\
&\left.\sum_{n=0}^{N} \frac{\omega}{c_{s}} \cos \left(\frac{n \pi}{\alpha} \theta\right) \cdot\left(C_{n} J^{\prime}\left(\frac{n \pi}{\alpha}, \frac{\omega r}{c_{s}}\right)\right)\right|_{r=R}
\end{aligned}
$$

where primes indicate differentiation with respect to $r$ (i.e., $d(..) / d r$ ). By letting reflected modes $L=N$. These continuity equations can be integrated through the angle of the wedge after multiplying them with a set of weight functions. By this way, it leads to a set of algebraic equations ( $3 \mathrm{~N}$ equations) in which the coefficients, $B_{l}, C_{n}$, and $D_{n}$ can be determined after setting intensity of the incident wave, $A_{i}$, to be one.

\section{RESULTS AND DISCUSSION}

A semi-infinite plate of a homogeneous, isotropic, linearly elastic material is considered. The elastic plate is made of stainless steel and has a density and a shear modulus of $7.8 \mathrm{~g} / \mathrm{cm}^{3}$ and $0.81 \times 10^{11} \mathrm{~Pa}$, respectively.

A time-harmonic SH wave propagates in the plate and is incident upon the inclined end of the plate. Since the end of the plate is considered to be stress free, the wave scattering problem reduces to determining the formed reflected wave field. Only the traveling modes have the capability of carrying the incident wave energy back into the plate. These traveling modes are supplemented by a series of end modes, which are associated with purely imaginary wave numbers, in order to satisfy a traction-free end conditions. The number of modes, $\mathrm{N}$, in the wave expansion function has been chosen to be 15 modes as to include all the propagating and non-propagating ones. The accuracy of the results is determined by studying the error norm in the energy balance.

In order to understand wave scattering, a single $\mathrm{SH}$ mode is incident on the free end of the plate and reflected SH modes are examined.. Two cases are considered; in the first, the zeroth symmetric mode is incident on the edge at selected frequencies and in the other, the first antisymmetric mode is incident on the edge at the same range of frequencies. The results are presented in terms of the energy content of the reflection modes for the two cases.

The inclined free end of the plate is allowed to take various angles of inclination. Figures 2 and 3 show the energy ratio $\left(E_{n} / E_{\text {total }}\right)$ of the reflected SH modes from the incidence of the zeroth $\mathrm{SH}$ mode over the full range of angles of inclination as functions of the normalized frequencies, $\Omega=(2 \omega \mathrm{h} / \pi \mathrm{c})$, of 1.5 . 


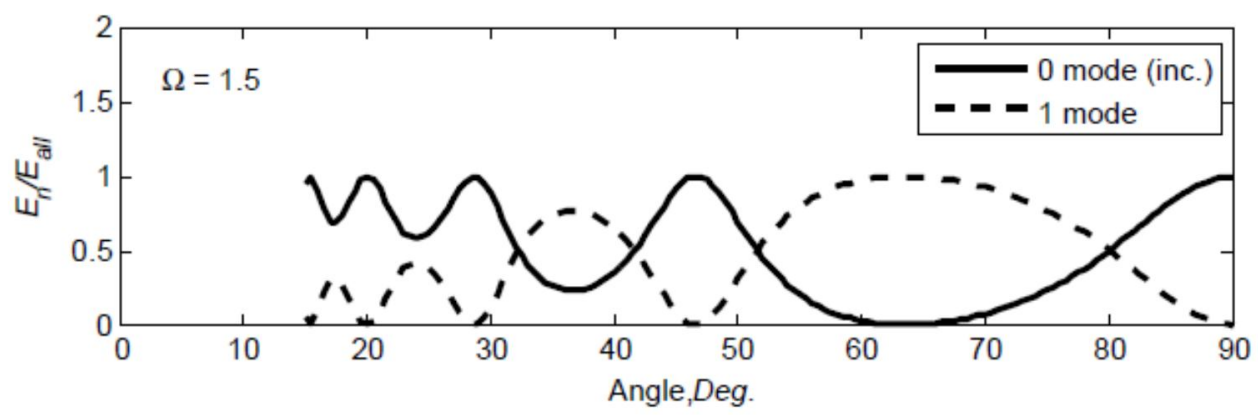

Fig. 2: The energy ratio $\left(E_{n} / E_{\text {total }}\right)$ of the reflected $\mathrm{SH}$ modes (symmetric).

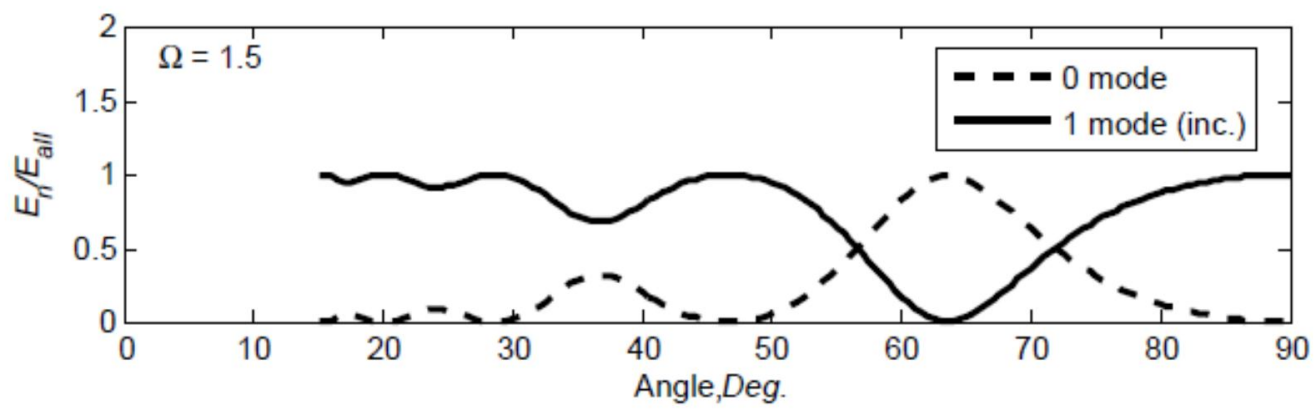

Fig. 3: The energy ratio $\left(E_{n} / E_{\text {total }}\right)$ of the reflected SH modes (anti-symmetric).

Figures 4 and 5 show the energy ratio $\left(E_{n} / E_{\text {total }}\right)$ of the reflected SH modes from the incidence of the zeroth SH mode over the range the normalized frequencies from 1 up to 2 . Figures 6 and 7 show corresponding results, $\left(E_{n} / E_{\text {total }}\right)$, for the case when the first SH mode is incident on the free edge.

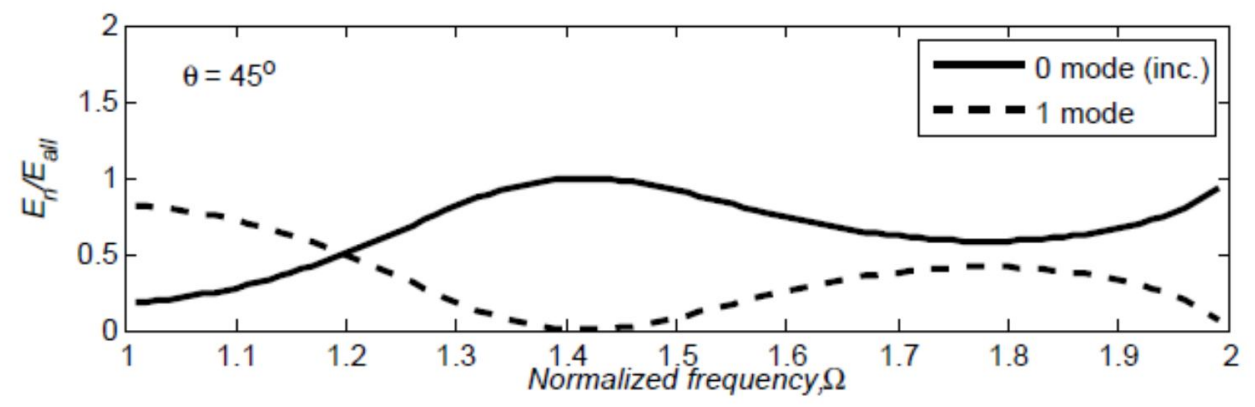

Fig. 4: The energy ratio $\left(E_{n} / E_{\text {total }}\right)$ of the reflected SH modes (symmetric). 


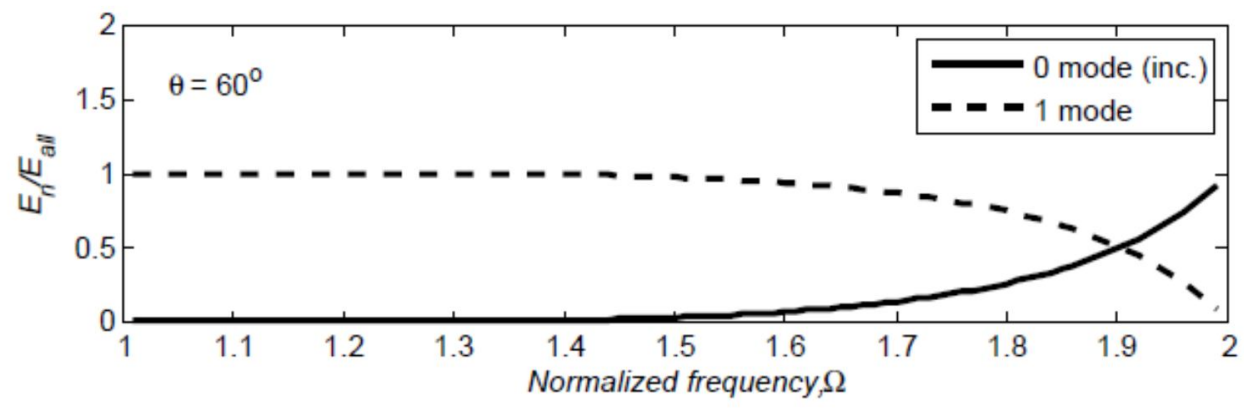

Fig. 5: The energy ratio $\left(E_{n} / E_{\text {total }}\right)$ of the reflected $\mathrm{SH}$ modes (symmetric).

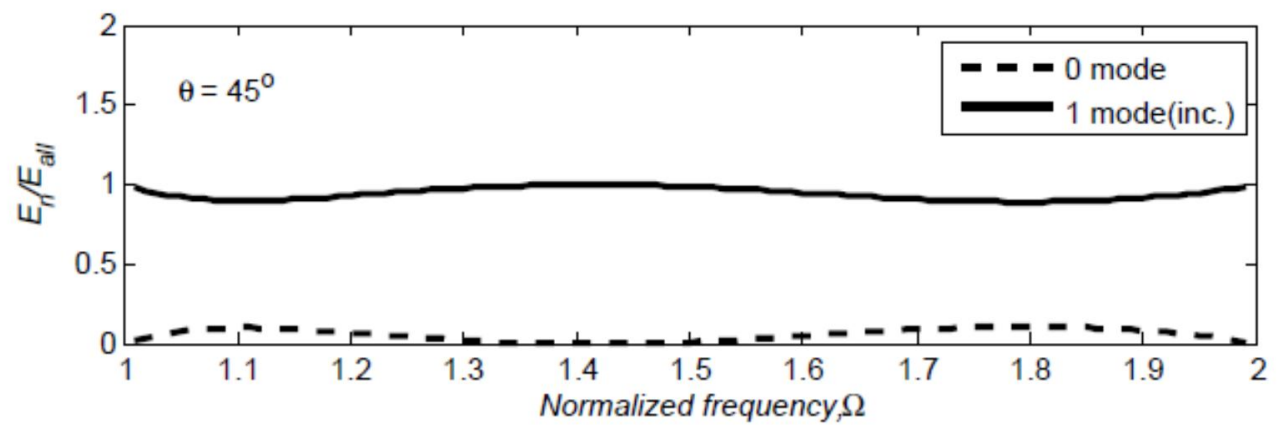

Fig. 6: The energy ratio $\left(E_{n} / E_{\text {total }}\right)$ of the reflected $\mathrm{SH}$ modes (anti-symmetric).

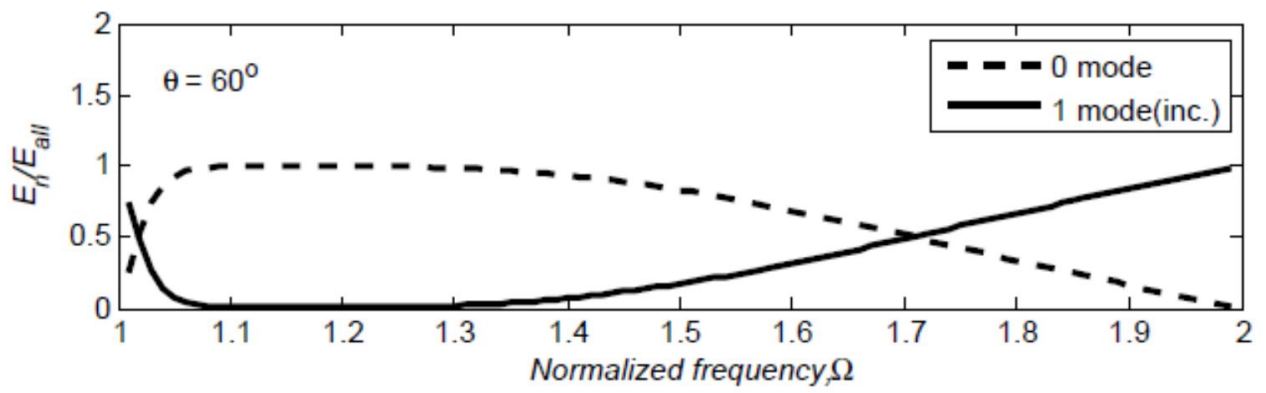

Fig. 7: The energy ratio $\left(E_{n} / E_{\text {total }}\right)$ of the reflected $\mathrm{SH}$ modes (anti-symmetric).

From the presented figures, one can report the following observations:

1. Mode conversion of SH modes occurs if the end of the plate is tapered at low normalized frequency (e.g., $\Omega=1.5$, where two propagating modes only) and for certain inclination angles.

2. At certain inclination angles, total (or most of) reflection energy is carried by the-incident mode itself, which increases the possibility of standing wave formation. 
3. There are, however, other inclination angles that leads to the formation of reflected fields comprised of non-incident modes. Hence, the ultrasonic field in the plate becomes multimodal in nature.

\section{CONCLUDING REMARKS}

The study reported here deals with a single incident mode only. These curves of the reflection coefficients may be very useful in practical nondestructive testing. In an actual experiment the incident disturbance will be due to a time dependent source. Then the field, in the frequency domain, will be made of multiple propagating modes. Using the outcome results, in terms of reflection coefficients, it would be possible to find the total scattered field by summing up the modal contributions. In addition, the reported results could be used as reference information base as a perfectly edged blade with no cracks or defects. Also, this study shows that there are some critical angles of inclination in which the incident mode (symmetric or anti-symmetric is totally reflected in an inversely fashion. In other words, an incident symmetric mode reflected into an anti-symmetric mode and vice versa.

\section{ACKNOWLEDGEMENT}

The authors would like to acknowledge the support offered by King Fahd University of Petroleum and Minerals.

\section{REFERENCES}

[1] H. A. Sabbagh and T. F. Krile, "Finite element analysis of elastic waves scattering from discontinuities". Ultrason Symp. Proc.,pp $216,1973$.

[2] Z. Abduljabbar, S. K. Datta, and A. H. Shah, "Diffraction of horizontaly polarized shear waves by normal cracks in a plate", J.Appl. Phys., Vol. 52, pp 461,1983.

[3] M. Koshiba, H .Morita, and M. Suzuki, "Finite element analysis of discontinuity problem of SH modes in an elastic plate-wave guide", Electronic. Lett., Vol. 17, pp 4801981.

[4] S. K. Datta, A. H. Shah, and C. M. Fortunko, "Diffraction of medium and long wavelength horizontaly polarized shear waves by edge crack", J. Appl. Phys., Vol 53, pp 2895, 1982.

[5] C. M. Fortunko, R. B. King, and M. Tan, "Nondestructive evaluation of planar defects using low-frequency shear horizontal waves", J. Appl. Phys., Vol. 55, pp 34501982.

[6] N. Wilkie-Chancellier, H. Duflo, A. Tinel, and J. Duclos, "Energy Balance in the Conversion of a Lamb Wave at a Bevelled Edge", Acta Acustica united with Acustica 90-1, pp. 77-84, 2004.

[7] N. Wilkie-Chancellier, H. Duflo, A. Tinel, and J. Duclos, "Experimental study and signal analysis in the Lamb wave conversion at a bevelled edge", Ultrasonics 42-1, pp. 377-381, 2004.

[8] N. Wilkie-Chancellier, H. Duflo, A. Tinel, and J. Duclos, "Numerical description of the edge mode at the beveled extremity of a plate", Journal of the Acoustical Society of America 117-1, pp. 194-199, 2005.

[9] J. M. Galán and R. Abascal, , "Lamb mode conversion at edges. A hybrid boundary elementfinite-element solution", Journal of the Acoustical Society of America 117-4I, pp. 1777-1784. 2005. 